\title{
Quick Recovery of Renal Alterations and Inflammatory Activation after a Marathon
}

\author{
Nayara Panizo González ${ }^{a}$ b Javier Eduardo Reque Santivañez ${ }^{c}$ \\ Barbara Hernando Fuster $^{\mathrm{b}}$ Eladio J. Collado Boirab Ignacio Martinez- \\ Navarrod,e Óscar Chiva Bartoll $^{f}$ Carlos Hernando Domingo ${ }^{f, g}$ \\ ${ }^{a}$ Hospital Universitario Clínico de Valencia, Valencia, Spain; ${ }^{b}$ Department of Health Sciences, Jaume I University, \\ Castellón, Spain; ' ${ }^{C}$ General Hospital of Castellón, Castellón, Spain; ${ }^{d}$ Sports Health Unit, Vithas-Nisa 9 de Octubre \\ Hospital, Valencia, Spain; ${ }^{~}$ Department of Physical Education and Sport, University of Valencia, Valencia, Spain; \\ fDepartment of Education, Jaume I University, Castellón, Spain; ${ }^{9}$ Sports Service, Jaume I University, Castellón, Spain
}

\section{Keywords}

Acute kidney injury - Marathon - Kidney damage resolution . Inflammatory activation $\cdot$ Microalbuminuria

\begin{abstract}
Purpose: We evaluated the incidence of acute kidney injury in a cohort of marathon participants. Methods: We conducted a prospective observational study focused on evaluating the incidence of kidney damage after a marathon, and its evolution in the first $48 \mathrm{~h}$ after the marathon in 88 runners who completed the Valencia Marathon. Results: From the 88 participants, 42 (48.28\%) presented with acute kidney injury, mainly grade 1 (95.20\%). Microscopic haematuria was observed in 29 runners (33\%). Levels of interleukin 6, leukocytes, and neutrophils were markedly increased at the marathon's finish line. Conclusions: Our results confirmed that there are slight transient changes in glomerular filtration rate and inflammatory activation after a marathon.
\end{abstract}

This study is enrolled in the ClinicalTrails.gov database, with the code No. NCT03155633 (www.clinicaltrials.gov).

\section{KARGER}

(c) 2019 S. Karger AG, Basel

E-Mail karger@karger.com

www.karger.com/kdd

\section{Introduction}

Since the study of Behrman [1] in 1941, it is well known that the physical stress of marathon running is associated with renal function alterations and urinary abnormalities. In the following years, several observational studies evaluated the impact of exhaustive physical exercise on the pathophysiology of renal injury and failure [2]. However, most of them assumed the classic dogma, which stated that the biochemical alterations observed in runners are the consequence of skeletal muscle destruction and dehydration caused by physical stress; the possibility of having intrinsic renal damage was ignored. Consequently, any research represents a step forward in elucidating the specific cause of pathological urinary changes.

Since long-distance races have substantially increased in popularity in recent years [3,4], a renewed interest in understanding the exercise-related mechanisms altering renal function has emerged. Recently, 2 independent studies showed (by measuring novel biomarkers of kidney damage) a clear impact of high-intensity exercise on renal function, which seems to be independent of both dehydration and skeletal muscle destruction $[5,6]$. The results of both studies pointed out the key role of system- 
ic inflammation in kidney damage. Systemic inflammation has been observed in marathon runners independent of renal function [7-9].

In this study, we evaluated the incidence of acute kidney injury (AKI) after marathon running in a cohort of 88 individuals, and the evolution of this alterations $48 \mathrm{~h}$ after the marathon.

\section{Material and Methods}

\section{Sample Subset Selection and Study Design}

All participants of the Valencia Marathon 2016 received an invitation by e-mail to participate in this study. Two informative seminars were organized to fully explain the study design (aims, protocol, hypothesis, etc.) to those individuals who accepted the invitation $(n=456)$. A total of 105 recreational marathon runners were selected to participate in this study, according to the following inclusion criteria: (1) an age between 30 and 45 years; (2) a body mass index (BMI) of between 16 and 24.99; (3) previous marathon experience, having a best-performance time of between 3 and $4 \mathrm{~h}$ for males and between 3:30 and 4:30 h for females; and (4) general good health, free from cardiac or renal disease and dyslipidemia. The selected participants had trained regularly during the last $6.76 \pm 3.19$ years and had finished an average of $3.24 \pm 3$ marathons. During their preparation for the Valencia Marathon, they trained an average of $4.78 \pm 0.89$ days a week, covering $62 \pm$ $13.52 \mathrm{~km}$.

On the 22nd of November 2016, a total of 95 individuals started the Valencia Marathon, 88 of whom (74 males and 14 females) crossed the finish line and were thus included in the study.

Prior to starting the study, all subjects performed a cardiopulmonary exercise test on a treadmill (pulsar ${ }^{\circledR} 3 \mathrm{p}, \mathrm{h} / \mathrm{p} /$ cosmos Sports and Medical GmbH, Nussdorf-Traunstein, Germany) until exhaustion. Breath-by-breath gas exchange was measured by the Jaeger MasterScreen ${ }^{\circledR}$ CPX gas analyzer to identify the first ventilatory threshold (VT1), the second ventilatory threshold (VT2), and the maximal oxygen consumption $\left(\mathrm{O}_{2 \max }\right)$ [10-12].

\section{Data Collection}

A web-based questionnaire was used to collect demographic and medical information, the training plan, and competition history.

The day before the marathon, anthropometric data (height and body mass) of all individuals was evaluated. Height was measured using a SECA 213 portable stadiometer (seca GmbH \& Co. KG, Hamburg, Germany). Body mass was assessed with light sport clothing and barefoot using a SECA 813 (seca GmbH \& Co. KG). BMI was then calculated. For the estimation of water balance, body mass was also measured both $1 \mathrm{~h}$ before the marathon start and after crossing the finish line. A medical examination was carried out by a nephrology specialist to assess dehydration data (skin and mucous dryness and skin-fold thickness). Hydration during the marathon was recorded in a personal interview of subjects.

Collection points of blood and urine samples as well as the biochemical markers analyzed in these samples are summarized in Table 1. Blood samples were taken from runners' antecubital veins by venipuncture using BD Vacutainer PST II tubes, centrifuged at
3,500 rpm for $10 \mathrm{~min}$, and transported to a hospital in Valencia at $4^{\circ} \mathrm{C}$ for biochemical analysis. The impact of modifications in plasma volume due to dehydration was taken into account according to the recommendations of Kargotich et al. [13]. To this end, the biochemical results collected at the post-marathon time points were adjusted with the method of Dill and Costill [14], using haematocrit and haemoglobin to determine the magnitude of plasma volume changes after the race in each participant $[14,15]$. The Chronic Kidney Disease Epidemiology Collaboration (CKD-EPI) equation was used to calculate the glomerular filtration rate (GFR) [16].

\section{Definition of AKI}

Acute Kidney Injury Network (AKIN) international criteria were applied. Grade 1 AKIN is defined as a sudden reduction of renal function determined by an absolute increase in serum creatinine of at least $0.3 \mathrm{mg} / \mathrm{dL}$ within $48 \mathrm{~h}(\geq 26.4 \mu \mathrm{mol} / \mathrm{L})$, or at least $50 \%$ (1.5-fold) from baseline [17]. Grade 2 AKIN is considered when serum creatinine levels increase $>200-300 \%$ from baseline [17]. Grade 3 AKIN represents an increase in serum creatinine $>300 \%$ from baseline, or a level of $\geq 4 \mathrm{mg} / \mathrm{dL}$ with an acute increase of at least $0.5 \mathrm{mg} / \mathrm{dL}$. As it was not possible to collect the urine output, this was not taken into account. Haematuria is distinguished by the abnormal presence of red blood cells ( $\geq 5$ red blood cells per high-power field) in the urine [18].

\section{Statistical Analysis}

The Kolmogorov-Smirnov test was used to test for the normality of data distribution. Mean and standard deviation [SD] for normally distributed continuous variables, median and interquartile range [IQR] for non-normally distributed continuous variables, and $n(\%)$ for categorical variables were used to report study data.

For categorical variables, McNemar's test was applied to compare data from different collection points within the same individual or group, while the $\chi^{2}$ test was used to evaluate differences between recovery groups.

For normally distributed continuous variables, repeated-measures ANOVA was used to compare data from different collection points within the same individual or group. Bonferroni's post hoc test was used for pairwise comparisons. For non-normally distributed continuous variables, Friedman's test was applied to compare data from different collection points within the same individual.

Statistical analysis was done using SPSS software v23, and twosided $p$ values $<0.05$ were considered statistically significant.

\section{Results}

The information collected from the 88 individuals included in this clinical trial is summarized in Table 1.

Forty-two of 88 participants (48.28\%) presented with AKI after the marathon (Table 2). Nobody exhibited grade $3 \mathrm{AKI}$. We note that, in this case, statistical analyses were done using data obtained from 87 participants, since the blood sample of 1 participant at the finish line collection point was not available. We did not find any clinical (BMI, hydration status, weight loss, age, and sex) or ana- 
Table 1. Baseline characteristics of 88 individuals included in this clinical trial

\begin{tabular}{lc}
\hline Age, years & $38.68 \pm 3.60$ \\
Male & $74(84.09)$ \\
BMI & $22.91 \pm 1.62$ \\
Hypertension & $1(1.14)$ \\
Type 1 diabetes & $1(1.14)$ \\
Smoking & $5(5.70)$ \\
Use of non-steroidal anti-inflammatory & $14(15.90)$ \\
$\quad$ drugs & \\
Number of days resting after marathon & $6.5(3-7)$ \\
$\quad$ running & $66(79.51)$ \\
Use of isotonic drinks during marathon & $1.50(1.00-2.00)$ \\
$\quad$ running & $2.12 \pm 0.87$ \\
Bluid intake during marathon munning, L & $50(63.30)$ \\
Skin and mucous dryness & $15(18.75)$ \\
Skin-fold thickness & $20(23.81)$ \\
Number of cramps during the marathon & $214.79 \pm 20.83$ \\
Average marathon time, min & \\
\hline
\end{tabular}

Data is presented as mean \pm SD for normally distributed continuous variables, median (interquartile range) for nonnormally distributed continuous variables, and $n(\%)$ for categorical variables.

lytical (CRP, Il-6, GFR, etc.) statistically significant differences between AKI and non-AKI participants.

Remarkably, haemoglobin was the only marker whose mean values before the marathon and at the finish line were not significantly different $(p>0.05)$ (Table 3$)$. Sodium levels in the urine were considerably decreased at the finish line collection point. Indeed, we observed alterations related to transient changes in glomerular filtration, such as the increase of the albumin/creatinine ratio in the urine and the percentage of runners with haematuria.

The blood analyses also showed a notable reduction of the GFR after crossing the finish line. After $24 \mathrm{~h}$, these GFR values returned to normal (Fig. 1). However, a slight, but significant, decrease in GFR was also observed at the 48-h post-marathon collection point. Although changes in GFR levels were not clinically significant (i.e., they were within the normal range) at $48 \mathrm{~h}$, this reduction suggests the importance of maintaining intensive oral hydration, not only during the first $24 \mathrm{~h}$ post-marathon when the protective mechanism of thirst is more intense, but also until at least $48 \mathrm{~h}$ after racing with vigorous physical effort.

We followed up all participants until $144 \mathrm{~h}$. At this time point, all of them had recovered their basal GFR,
Table 2. Incidence of acute renal failure after running the marathon

\begin{tabular}{lc}
\hline Absence of AKI & $45(51.72)$ \\
Presence of AKI & $42(48.28)$ \\
$\quad$ Grade 1 & $40(95.24)$ \\
$\quad$ Grade 2 & $2(4.76)$ \\
Presence of haematuria & $29(33.00)$
\end{tabular}

Data is presented as $n(\%)$. AKI, acute kidney injury.

with no differences between those in whom it had decreased and those in whom it had not.

\section{Discussion}

The popularity of studying mechanisms altering renal function after high-intensity exercise has risen considerably over the last few years $[19,20]$. This prospective cohort study recruited a considerable number of volunteer marathon runners compared to previously published studies $[6,21]$.

High-intensity exercise was confirmed to impact on renal function, since approximately half of the runners (48.28\%) presented with AKI after the marathon, and microscopic haematuria was observed in one-third (33.00\%). Indeed, the level of microalbuminuria reached was considerably high compared to normal conditions $(59.78 \pm$ 89.50 vs. $<10 \mathrm{mg} / \mathrm{dL}$ ).

Our results were consistent with the observations by McCullough et al. [5] which revealed that $40 \%$ of 25 subjects presented with acute renal failure after running a marathon. However, this percentage was considerably higher in a very recently published study ( $82 \%$ of 22 runners showed renal failure) [6]. The slight difference between McCullough et al. [5] and our study may be due to differences in the average completion time of the runners $(256.2 \pm 43.5$ vs. $214 \pm 20.8 \mathrm{~min})$, assuming that a faster marathon time may be associated with a higher physical effort, and, therefore, with a greater impact on runners' health status. Nevertheless, this hypothesis is utterly incongruous with the results of the study by Mansour et al. [6], which found that most runners presented with AKI despite having a slower marathon time of $240.12 \pm 38.4$ $\min$. Note that none of the previous studies adjusted the biomarker levels collected at the finish line based on blood volume loss as should be done [22]. Our non-adjusted results were practically identical with those previ- 
Fig. 1. Evolution of glomerular filtration rate alterations after running a marathon. A comparison of data from 4 collection points was performed. Data collected from the start line to $48 \mathrm{~h}$ post-marathon running was used. FL, finish line; SL, start line. ${ }^{*} p<0.05$; $^{* *} p<0.01$; $^{* * *} p<0.001$.

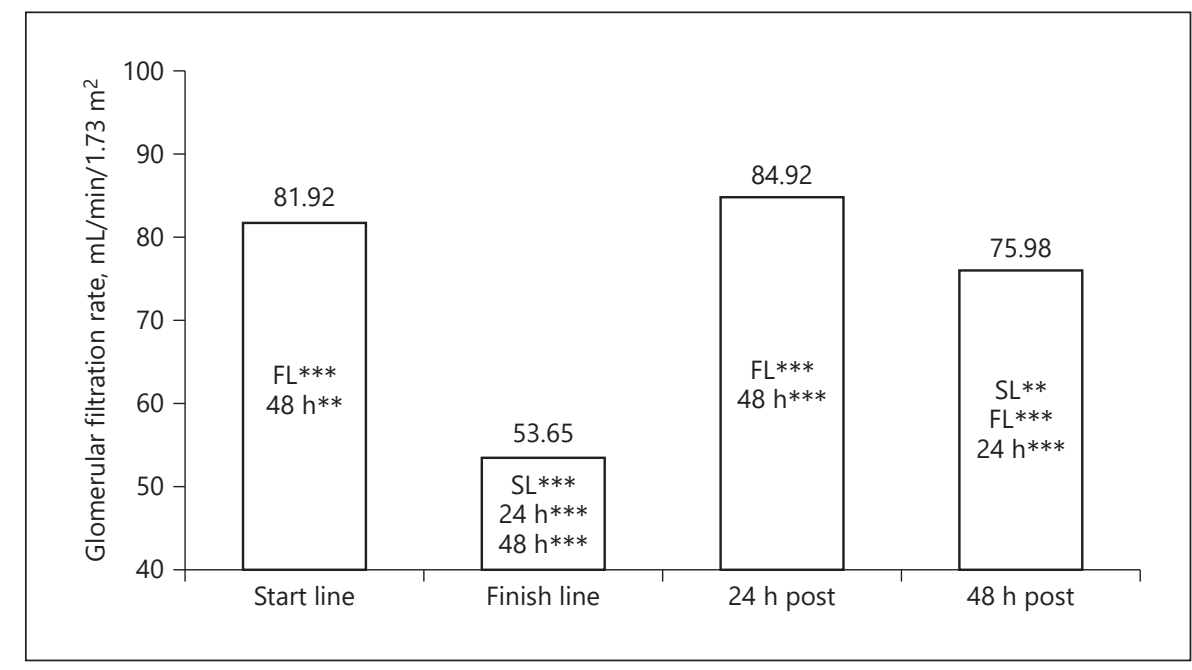

Table 3. Data of subjects at the 4 collection time points

\begin{tabular}{|c|c|c|c|c|c|c|}
\hline$N$ & Characteristic & At the start line & At the finish line & $24 \mathrm{~h}$ post-marathon & $48 \mathrm{~h}$ post-marathon & $\begin{array}{l}\text { Overall } \\
p \text { value }\end{array}$ \\
\hline 82 & Haemoglobin, g/dL & $14.69 \pm 0.95$ & $14.82 \pm 1.02$ & $13.70 \pm 0.92^{\mathrm{b}}$ & $13.88 \pm 0.84^{\mathrm{b}}$ & $<0.001$ \\
\hline 82 & Leukocytes, $10^{6} / \mu \mathrm{L}$ & $7.14 \pm 1.75$ & $17.39 \pm 3.94^{\mathrm{b}}$ & $7.61 \pm 1.50$ & $7.27 \pm 1.30$ & $<0.001$ \\
\hline 82 & Neutrophils, $10^{6} / \mu \mathrm{L}$ & $4.11 \pm 1.32$ & $14.50 \pm 3.67^{\mathrm{b}}$ & $4.22 \pm 1.20$ & $3.879 \pm 0.86$ & $<0.001$ \\
\hline 84 & $\mathrm{CPR}, \mathrm{mg} / \mathrm{dL}$ & $0.04(0.02-0.05)^{\mathrm{b}}$ & $4.85(4.68-5.01)^{\mathrm{b}}$ & $1.22(0.90-1.60)^{\mathrm{b}}$ & $0.67(0.47-0.82)^{\mathrm{b}}$ & $<0.001$ \\
\hline 85 & IL-6, pg/mL & $3.10(2.18-5.60)$ & $83.75(64.72-130.14)$ & n.a. & n.a. & $<0.001$ \\
\hline 81 & Creatinine, mg/dL & $0.96(0.80-1.10)$ & $1.31(1.17-1.47)^{\mathrm{b}}$ & $0.94(0.80-1.00)$ & $1.01(0.90-1.10)^{\mathrm{b}}$ & $<0.001$ \\
\hline 81 & GFR, $\mathrm{mL} / \mathrm{min} / 1.73 \mathrm{~m}^{2}$ & $81.92(66.13-98.75)$ & $53.65(45.12-60.53)^{\mathrm{b}}$ & $84.92(68.93-102.28)$ & $75.98(63.62-94.58)^{\mathrm{b}}$ & $<0.001$ \\
\hline 82 & Urea, mg/dL & $34.00(29.00-40.00)^{\mathrm{b}}$ & $42.32(37.60-46.66)^{\mathrm{b}}$ & $38.00(33.00-45.00)^{\mathrm{b}}$ & $35.00(30.75-41.25)^{\mathrm{b}}$ & $<0.001$ \\
\hline 82 & $\mathrm{CK}, \mathrm{UI} / \mathrm{L}$ & $136.00(106.00-183.00)^{\mathrm{b}}$ & $340.27(268.01-432.44)^{\mathrm{b}}$ & $1,043.00(561.00-1,742.00)^{\mathrm{b}}$ & $514.00(304.00-816.00)^{\mathrm{b}}$ & $<0.001$ \\
\hline 81 & $\mathrm{LDH}, \mathrm{UI} / \mathrm{L}$ & $175.00(160.00-197.00)^{\mathrm{b}}$ & $306.18(274.54-362.06)^{\mathrm{b}}$ & $229.00(203.75-261.75)^{\mathrm{b}}$ & $247.50(214.00-281.25)^{\mathrm{b}}$ & $<0.001$ \\
\hline 82 & Uric acid, mg/dL & $4.50(4.00-5.00)$ & $5.53(4.83-6.27)$ & $4.70(4.10-5.70)$ & $4.70(4.00-5.23)$ & $<0.001$ \\
\hline \multicolumn{7}{|c|}{ Urinary parameters } \\
\hline 81 & Microalbuminuria, $\mathrm{mg} / \mathrm{dL}$ & $002.25(1.50-5.23)^{c}$ & $032.35(13.88-58.33)^{c}$ & n.a. & $1.60(0.70-2.90)^{c}$ & $<0.001$ \\
\hline 88 & Urinary sodium, mEq/L & $089.50(60.25-114.75)$ & $039.50(21.00-62.25)^{c}$ & n.a. & $86.00(58.25-134.75)$ & $<0.001$ \\
\hline 81 & ACR, mg/g & $001.95(1.20-2.88)^{\mathrm{c}}$ & $017.80(11.00-28.88)^{c}$ & n.a. & $1.50(1.00-2.40)^{\mathrm{c}}$ & $<0.001$ \\
\hline 88 & Microhaematuria & $0000.6(6.80 \%)$ & $000.29(33.00 \%)^{c}$ & n.a. & $3(3.40 \%)$ & $<0.001$ \\
\hline
\end{tabular}

Data is presented as mean \pm SD for normally distributed continuous variables, median (interquartile range) for non-normally distributed continuous variables, and $n$ (\%) for categorical variables. Data from blood samples was adjusted following Dill and Costill's method [14]. N, number of runners; CK, creatine kinase; IL, interleukin; LDH, lactate dehydrogenase; CPR, C-reactive protein; GFR, glomerular filtration rate (calculated by the CKD-EPI equation [16]); ACR, albumin/creatinine ratio; n.a., not available. Conversion factors for units: serum creatinine in $\mathrm{mg} / \mathrm{dL}$ to $\mu \mathrm{mol} / \mathrm{L}, \times 88.4$; urea nitrogen in $\mathrm{mg} / \mathrm{dL}$ to $\mathrm{mmol} / \mathrm{L}, \times 0.357$; uric acid in $\mathrm{mg} / \mathrm{dL}$ to $\mu \mathrm{mol} / \mathrm{L}, \times 59.48$.

a $p$ values were calculated with McNemar's test, repeated-measures ANOVA, and Friedman's test for categorical variables, normally, and non-normally distributed continuous variables, respectively. Pairwise comparisons were done by applying the Bonferroni and Wilcoxon-Mann-Whitney post hoc tests for normally and non-normally distributed continuous variables, respectively.

b Statistically significantly different from the other 3 collection points.

c Statistically significantly different at 2 collection points.

ously published (data not shown). Altogether, our observations may mainly differ from previous published because of the sample size. Having a larger sample size produces results that are more representative. Therefore, the incidence of renal alterations after marathon running could be more accurately studied in this study.

Like in McCullough et al. [5] and Mansour et al. [6], most of the renal alterations were grade 1 . Renal alterations were confirmed to be completely recovered in a short period of time $[5,6,23]$. In contrast to previous clas- sical studies [24-26], none of the runners displayed severe renal failure, severe rhabdomyolysis, or multiorgan failure, and there were no deaths after the marathon. We speculate that, nowadays, runners are better physically prepared and informed about the risks of dehydration.

Unlike serum creatinine (the level of which may be modified by the breakdown of muscular fibres), serum creatine kinase (CK) did not reach its maximum level at the finish line but rather at the 24 -h post-marathon collection point, when the creatinine level had returned to 
normal. This observation, also shown in a cohort study of 34 marathoners [27], confirmed that renal alterations are not a mere "biochemical illusion" subsequent to the release of muscular intracellular components as a result of the breakdown of muscle fibres. This is also supported by McCullough et al. [5] and Mansour et al. [6], who revealed an increased level of tubular kidney damage-specific biomarkers that appears to be independent of muscle mass and dehydration status, such as neutrophil gelatinase-associated lipocalin (NGAL), kidney injury molecule-1 (KIM-1), and cystatin C.

Even so, reaching the maximum CK level $24 \mathrm{~h}$ after the marathon, together with the fact that both uric acid and lactate dehydrogenase remained high with respect to baseline levels $48 \mathrm{~h}$ post-marathon, suggests that the reduction of GFR observed at the 48-h collection point may have been due to the late arrival of intracellular toxic waste to the kidney.

Additionally, as previously shown $[5,6]$, urinary sodium is reduced after running a marathon because, in response to the body fluid loss, a healthy kidney retains sodium and therefore water. As result, a small volume of more concentrated urine is excreted (the sodium is less diluted) [28]. Urine sodium levels were not associated either with the development of acute renal failure, or with the second GFR reduction observed during the post-marathon follow-up period.

Taking everything into account, neurohormonal mechanisms derived from inflammatory activity and/or oxidative stress may be involved in renal failure, since it appears that tubular injury is not the only cause of renal alterations observed in runners after a marathon. Indeed, we also found urinary alterations, such as microalbuminuria and microhaematuria, which are derived from alterations in glomerular permeability. The fact that alterations in glomerular permeability underlie most chronic kidney disorders, due to the accumulation of cardiovascular risk factors [29], highlights the importance of this assumption which should be confirmed by further specifically designed studies. The transient renal alterations found in marathoners (healthy individuals who did not present with cardiovascular risk factors or chronic renal disorders) may represent an excellent model (free of confounding variables) to study the role of inflammation and oxidative stress in renal autoregulatory mechanisms.

On the one hand, a fluid sequestration within the muscles and an imbalance between vasodilation and vasoconstrictor agents because of the endothelial dysfunction development derived from the release of myoglobin into the circulation have been shown during strenuous physical exercise [30]. Both conditions lead to the activation of the renin-angiotensin-aldosterone system (RAAS), and therefore to intrarenal vasoconstriction [30].

On the other hand, alterations in microvascular blood flow and leukocyte recruitment are clearly correlated through the ischemia/reperfusion phenomenon [31]. Under these circumstances, as confirmed by our results, reactive oxygen species (ROS) and inflammatory mediators increase leukocyte activation and recruitment. This, in turn, intensifies inflammation, endothelial activation, and vascular tone. Indeed, as shown in our marathon runners, the renal epithelium also generates interleukin 6 (IL-6), a cytokine that triggers the inflammatory cascade [32]. IL-6 is shown to aggravate kidney damage in rats $[33,34]$, and also in humans with ischaemic renal damage who are undergoing cardiac surgery [35].

Further studies are needed to deeply understand these inflammatory mechanisms as well as delineate the molecules that block the inflammatory cascade and oxidative stress. These chemicals may present a great therapeutic and preventive utility when the renal vasculature is compromised by the endothelial dysfunction that they cause. Some of them, such as $\mathrm{N}$-acetylcysteine [36], vitamin C [37], and acetaminophen [38], are already being used for the treatment of other indications, and they have a high safety profile. Indeed, they have been positively tested in animal models that reproduce the type of AKI observed in our subjects after marathon running. Future clinical studies in human models (i.e., healthy marathoners) that focuses on testing the effect of anti-inflammatory and anti-oxidative chemicals in renal failure prevention should be considered.

\section{Practical Applications and Conclusions}

In conclusion, we observed slight transient changes in GFR after marathon running as well as data that indicated glomerular structural damage (microalbuminuria and haematuria). However, our data suggests that the longterm repercussions of these in renal function are not foreseeable since such alterations were immediately normalized (all data returned to normal values at $24-48 \mathrm{~h}$ after the marathon). This is important information for coaches and runners.

According to our data, mechanisms of inflammatory origin involved in endothelial dysfunction could trigger the renal alterations observed after marathon running. These results can encourage researchers to develop clinical studies focused on testing the effect of anti-inflamma- 
tory and anti-oxidative chemicals for the prevention of AKI. A large-scale, prospective study evaluating biomarkers related to renal failure in athletes, such as NGAL and KIM-1, before (in the preparation phase) and after (in the recovery phase) competing is necessary to confirm our findings.

\section{Acknowledgements}

We are grateful to all the runners and volunteers who participated in this study and to the funding institutions: Trinidad Alfonso Foundation, Vithas NISA Hospital Foundation, and Correcaminos Sports Association.

\section{Statement of Ethics}

All individuals included were fully informed and gave their written consent to participate. The research was conducted according to the Declaration of Helsinki, and it was approved by the Research Ethics Committee of the Jaume I University of Castellon.

\section{Disclosure Statement}

The authors have no conflicts of interest to declare. The results presented in this paper have not been published previously as a whole or in part.

\section{References}

1 Behrman R. Urinary findings before and after a marathon race. N Engl J Med. 1941;225(20): $801-2$.

2 Thompson PD, Venero CV. A history of medical reports on the Boston Marathon: 112 years and still running. Med Sci Sports Exerc. 2009 Jun;41(6):1341-8.

3 El Helou N, Tafflet M, Berthelot G, Tolaini J, Marc A, Guillaume M, et al. Impact of environmental parameters on marathon running performance. PLoS One. 2012;7(5):e37407.

4 Ahmadyar B, Rüst CA, Rosemann T, Knechtle B. Participation and performance trends in elderly marathoners in four of the world's largest marathons during 2004-2011. Springerplus. 2015 Aug;4(1):465.

5 McCullough PA, Chinnaiyan KM, Gallagher MJ, Colar JM, Geddes T, Gold JM, et al. Changes in renal markers and acute kidney injury after marathon running. Nephrology (Carlton). 2011 Feb;16(2):194-9.

6 Mansour SG, Verma G, Pata RW, Martin TG, Perazella MA, Parikh CR. Kidney Injury and Repair Biomarkers in Marathon Runners. Am J Kidney Dis. 2017;70(2):252-261.

7 Sugama K, Suzuki K, Yoshitani K, Shiraishi K, Kometani T. Urinary excretion of cytokines versus their plasma levels after endurance exercise. Exerc Immunol Rev. 2013;19:29-48.

8 Suzuki K, Nakaji S, Yamada M, Liu Q, Kurakake S, Okamura N, et al. Impact of a competitive marathon race on systemic cytokine and neutrophil responses. Med Sci Sports Exerc. $2003 \mathrm{Feb} ; 35(2): 348-55$.

9 Jee H, Jin Y. Effects of prolonged endurance exercise on vascular endothelial and inflammation markers. J Sports Sci Med. 2012 Dec; 11(4):719-26.

10 Beaver WL, Wasserman K, Whipp BJ. A new method for detecting anaerobic threshold by gas exchange. J Appl Physiol (1985). 1986 Jun; 60(6):2020-7.
11 Skinner JS, McLellan TM. The transition from aerobic to anaerobic metabolism. Res Q Exerc Sport. 1980 Mar;51(1):234-48.

12 McLellan TM, Skinner JS. Submaximal endurance performance related to the ventilation thresholds. Can J Appl Sport Sci. 1985 Jun; 10(2):81-7.

13 Kargotich S, Goodman C, Keast D, Fry RW, Garcia-Webb P, Crawford PM, et al. Influence of exercise-induced plasma volume changes on the interpretation of biochemical data following high-intensity exercise. Clin J Sport Med. 1997 Jul;7(3):185-91.

14 Dill DB, Costill DL. Calculation of percentage changes in volumes of blood, plasma, and red cells in dehydration. J Appl Physiol. 1974 Aug;37(2):247-8.

15 Alis R, Sanchis-Gomar F, Primo-Carrau C, Lozano-Calve S, Dipalo M, Aloe R, et al. Hemoconcentration induced by exercise: Revisiting the Dill and Costill equation. Scand J Med Sci Sports. 2015 Dec;25(6):e630-7.

16 Levey AS, Stevens LA, Schmid CH, Zhang YL, Castro AF 3rd, Feldman HI, et al.; CKD-EPI (Chronic Kidney Disease Epidemiology Collaboration). A new equation to estimate glomerular filtration rate. Ann Intern Med. 2009 May;150(9):604-12.

17 Mehta RL, Kellum JA, Shah SV, Molitoris BA, Ronco C, Warnock DG, et al.; Acute Kidney Injury Network. Acute Kidney Injury Network: report of an initiative to improve outcomes in acute kidney injury. Crit Care. 2007; 11(2):R31.

18 Kiragu D, Cifu AS. Evaluation of Patients With Asymptomatic Microhematuria. JAMA. 2015 Nov;314(17):1865-6.

19 Oliveira RA, Sierra AP, Benetti M, Ghorayeb $\mathrm{N}$, Sierra CA, Kiss MA, et al. Impact of Hot Environment on Fluid and Electrolyte Imbalance, Renal Damage, Hemolysis, and Immune Activation Postmarathon. Oxid Med Cell Longev. 2017;2017:9824192.
20 Briviba K, Watzl B, Nickel K, Kulling S, Bös K, Haertel S, et al. A half-marathon and a marathon run induce oxidative DNA damage, reduce antioxidant capacity to protect DNA against damage and modify immune function in hobby runners. Redox Rep. 2005;10(6):325-31.

21 Vezzoli A, Dellanoce C, Mrakic-Sposta S, Montorsi M, Moretti S, Tonini A, et al. Oxidative Stress Assessment in Response to Ultraendurance Exercise: Thiols Redox Status and ROS Production according to Duration of a Competitive Race. Oxid Med Cell Longev. 2016;2016:6439037.

22 Ohira Y, Ito A, Ikawa S. Correction of water content and solute concentration in blood during hemoconcentration. J Appl Physiol. 1977 May;42(5):739-43.

23 Traiperm N, Gatterer H, Pariwat P, Burtscher $M$. Energy metabolism, liver and kidney function in adolescent marathon runners. Eur J Clin Invest. 2016 Jan;46(1):27-33.

24 Clarkson PM. Exertional rhabdomyolysis and acute renal failure in marathon runners. Sports Med. 2007;37(4-5):361-3.

25 Asserraji M, Benameur I, Maoujoud O, El Kharras A, Hajbi H, Filali K. Late care in marathon runs leading to exertional heat stroke with multiple organ failure. Asian J Sports Med. 2014 Jun;5(2):136-8.

26 MacSearraigh ET, Kallmeyer JC, Schiff HB. Acute renal failure in marathon runners. Nephron. 1979;24(5):236-40.

27 Bekos C, Zimmermann M, Unger L, Janik S, Hacker P, Mitterbauer A, et al. Non-professional marathon running: RAGE axis and ST2 family changes in relation to open-window effect, inflammation and renal function. Sci Rep. 2016 Sep;6(1):32315.

28 Bagshaw SM, Bennett M, Devarajan P, Bellomo R. Urine biochemistry in septic and nonseptic acute kidney injury: a prospective observational study. J Crit Care. 2013 Aug;28(4): 371-8. 
29 Salmon AH, Ferguson JK, Burford JL, Gevorgyan $\mathrm{H}$, Nakano D, Harper SJ, et al. Loss of the endothelial glycocalyx links albuminuria and vascular dysfunction. J Am Soc Nephrol. 2012 Aug;23(8):1339-50.

30 Panizo N, Rubio-Navarro A, Amaro-Villalobos JM, Egido J, Moreno JA. Molecular Mechanisms and Novel Therapeutic Approaches to Rhabdomyolysis-Induced Acute Kidney Injury. Kidney Blood Press Res. 2015;40(5): 520-32.

31 Linas SL, Shanley PF, Whittenburg D, Berger E, Repine JE. Neutrophils accentuate ischemia-reperfusion injury in isolated perfused rat kidneys. Am J Physiol. 1988 Oct;255(4 Pt 2):F728-35
32 Bonventre JV, Zuk A. Ischemic acute renal failure: an inflammatory disease? Kidney Int. 2004 Aug;66(2):480-5.

33 Kielar ML, John R, Bennett M, Richardson JA, Shelton JM, Chen L, et al. Maladaptive role of IL-6 in ischemic acute renal failure. J Am Soc Nephrol. 2005 Nov; 16(11):3315-25.

34 Patel NS, Chatterjee PK, Di Paola R, Mazzon E, Britti D, De Sarro A, et al. Endogenous interleukin-6 enhances the renal injury, dysfunction, and inflammation caused by ischemia/reperfusion. J Pharmacol Exp Ther. 2005 Mar;312(3):1170-8.

35 Zhang WR, Garg AX, Coca SG, Devereaux PJ, Eikelboom J, Kavsak P, et al.; TRIBE-AKI Consortium. Plasma IL-6 and IL-10 Concentrations Predict AKI and Long-Term Mortality in Adults after Cardiac Surgery. J Am Soc Nephrol. 2015 Dec;26(12):3123-32.
36 Kim JH, Lee SS, Jung MH, et al. N-acetylcysteine attenuates glycerol-induced acute kidney injury by regulating MAPKs and Bcl-2 family proteins. Nephrol Dial Transplant. 2010;25(5):1435-43.

37 Groebler LK, Wang XS, Kim HB, Shanu A, Hossain F, McMahon AC, et al. Cosupplementation with a synthetic, lipid-soluble polyphenol and vitamin $\mathrm{C}$ inhibits oxidative damage and improves vascular function yet does not inhibit acute renal injury in an animal model of rhabdomyolysis. Free Radic Biol Med. 2012 May;52(9):1918-28.

38 Boutaud O, Roberts LJ 2nd. Mechanismbased therapeutic approaches to rhabdomyolysis-induced renal failure. Free Radic Biol Med. 2011 Sep;51(5):1062-7. 